\title{
Anomalous stress reactions in patients suffering from depression and anxiety
}

\author{
HARRY BRIERLEY AND ROBIN JAMIESON \\ From the Professorial Unit of Psychological Medicine, General Hospital, Newcastle upon Tyne
}

\begin{abstract}
SYNOPSIS An experiment is reported in which the forearm muscle blood flow of a group of patients with mixed depressions is compared with that of a group of patients with anxiety states. The blood flow was measured under relaxed conditions and during the presentation of a noise 'stress'. The measurements obtained under stressed conditions show that, while the blood flow of those with anxiety states fell with repetition of the stress noise, that of the depressive patients increased. A similar pattern was shown by the relaxed measurements but here the difference was not statistically significant. There was also suggestion that stress produced a fall in blood flow in depressive subjects and an increase in patients with anxiety states. These results tend to support the hypothesis that depressive patients show something akin to a freeze response to stress, while patients with anxiety states show an arousal response.
\end{abstract}

Many workers have considered the significance of physiological activity levels. Kelly and Walter (1968), for example, have made out a case for regarding forearm muscle blood flow as a measure of anxiety. Duffy (1962), concerned with the whole range of physiological indices, uses the term 'activation' as having similar meaning to 'arousal', 'energy mobilization', and 'excitation'. Lader and Matthews (1968) were more cautious and use the term 'arousal' in a 'non-theoretical way' so that anxiety is an aspect of over-arousal as compared with the simple alertness of moderate arousal. Precisely what aspects of behaviour are represented by changes in physiological indices are not well established and Lader's conservative use of the term 'arousal' appears to reflect best the significance of psychophysiological measurements in the present state of knowledge. Increases in these levels apparently brought about by a variety of 'stresses', such as noise, mental arithmetic, and emotion, have commonly been observed. There is a temptation to assume that psychophysiological values represent anxiety in some form, so that where subjects with agitated depression are shown to have higher levels of blood flow than those with retarded depression this has been interpreted as reflecting the higher anxiety component exhibited by the agitated depressives. However, as Kelly and Walter (1968) have shown, 'basal' forearm blood flow values are not very highly related to anxiety as measured by either clinical judgement or conventional test methods, correlations with these standards being of the order of less than $0 \cdot 25$. It seems, therefore, that despite significant differences in blood flow between various clinical groups as shown by Kelly these differences cannot be attributed to anxiety alone in any conventional sense of the term. Moreover the term 'stresser' in this context does not need to imply any discomfort, since similar increases can be brought about by relatively pleasant stimuli-for example, rhythmical music or erotic fantasies, which appear to affect the level of alertness of the subject.

Brierley (1969) reported an investigation of the forearm muscle blood flow of patients suffering from phobic anxiety. Lader and Wing (1964) had suggested that if it were found that phobic patients showed increasing arousal as a result of continued stress a positive feedback cycle might be produced. That is to say, the more aroused the subject became the greater would be the arousal effect of a given stimulus. Such a situation could well be the mechanism of phobic panics. The findings in Brierley's experiment were anomalous. Phobic patients showed a 


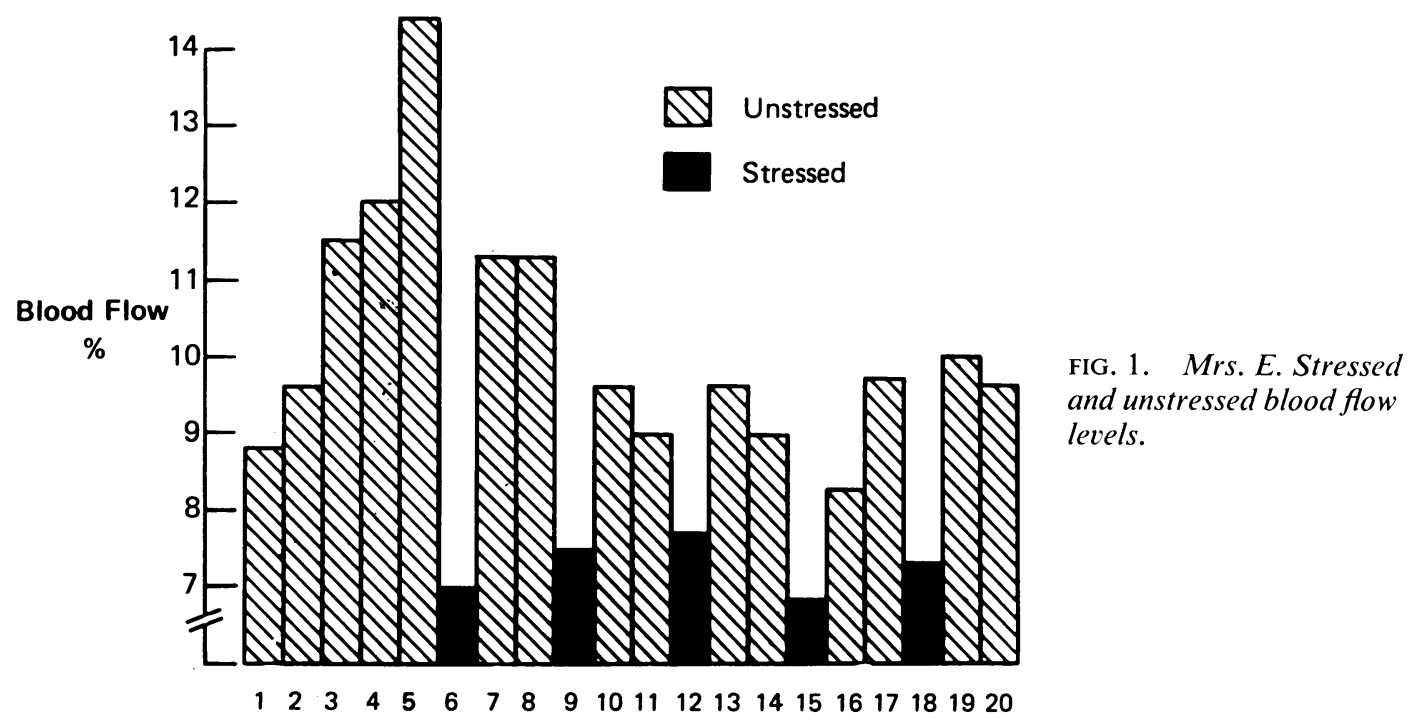

No. of Recording

smaller increase in forearm muscle blood flow as a result of exposure to a noise stimulus as a stresser than did normal subjects. Also repeated blood flow measurements showed a decrease with time and repetition of the stresser--that is, they showed habituation. Thus, the hypothesis of positive feedback was not supported by that experiment.

It might have been anticipated that a noise stresser would produce an increase in blood flow like that resulting from stressed mental arithmetic which Kelly (1966) has reported. This would be parallel with the positive arousal increases shown by Lader and Wing (1964) for the psychogalvanic reflex. However, a continuation of Brierley's investigation found a number of patients who exhibited an apparently anomalous fall in blood flow under experimental stress, compared with their relaxed levels. Three other factors seemed associated with this phenomenon. Firstly, these patients appeared to exhibit more depressed affect. Secondly, unlike the patients reported in Brierley (1969), the blood flow in these patients actually increased with time during the measurement session-that is, they became more aroused and did not habituate. Thirdly, a number of these patients became upset and continuation of the measurement appeared likely to give rise to panic.
A record demonstrating forearm blood flo measured during the presentation of a noise stresser, consistently below the relaxed level of blood flow is shown in Fig. 1. Here the stressed. readings have an average of $7.3 \% / \mathrm{min}$ compared with the relaxed level of $10.3 \% / \mathrm{n}$ no (This difference is statistically significant $\mathrm{a} \dot{\mathrm{s}}$ tested by the Mann-Whitney U Test).

This patient was a 44 year old woman whose diagnosis was 'a depressive illness in a woman oณ an obsessional personality who has a tendency to be rigid and anxiety prone'. She had been unde intermittent psychiatric care for over 12 years and initially she was believed to suffer from a recurrent endogenous depression. More recently? hypochondriacal preoccupations, complaints of defective eyesight, low back pain, and genito urinary problems, etc, had resulted in examina tions by numerous departments. She was rather aggressive lady who steadfastly refused to̊ accept herself as suffering from any psychiatric disability. At times she threatened suicide ancP demanded 'an operation for the pain'. She expressed dissatisfaction with all doctors who examined and treated her and refused to remain in hospital for treatment. Her husband, o builder's labourer, reported that her mood changed dramatically at times. Normally, she enjoyed going with him to a club and she liked 
playing the piano. When she was ill she fought with him, accused him of being alcoholic and a gambler. She would also believe she was clairvoyant and at times her suspicious behaviour caused family difficulties.

Figure 1 shows, in addition, the way that Mrs. E's forearm blood flow increased rapidly over the first five recordings in contrast with the way in which a normal record would demonstrate a fall in blood flow.

These observations were reported by Jamieson (1970). He postulated that the increased arousal response to a stress signal was akin to the familiar mobilization-for-flight reaction in fear, while the response of reduced arousal level was of the 'freeze' type. Such freeze behaviour in fear is familiar in the animal kingdom and, of course, even humans describe themselves as 'petrified' or 'frozen to the spot' in fear. However, there is little, if any, existing evidence of the existence of such freeze responses in humans but the evidence Jamieson presented seemed to illustrate a pattern of physiological stress response of this type.

The upshot of these observations was that it was decided to test two hypotheses:

1. That patients suffering from depression do not show the habituation of forearm muscle blood flow exhibited by patients with anxiety states but show an increase in flow during the measurement period.

2. That patients with anxiety states respond to a stress with an increase in forearm blood flow while depressives respond with a reduction in flow level.

\section{METHOD}

Forearm muscle blood flow is measured by occluding the venous return of blood from the forearm by a sphygmomanometer cuff fitted to the upper arm and measuring the increase in volume in the forearm. It is necessary to fit a cuff at the wrist to occlude the arterial flow to the hand, since the flow to the hand is primarily through the skin rather than muscle. The volume increase of the forearm is measured in a variety of ways. Most workers-for example, Kelly -have employed the waterbath plethysmograph which encases the forearm in a metal tank and hence largely immobilizes the patient. The technique employed by Whitney (1953) uses a mercury-inrubber strain gauge. This is a $0.5 \mathrm{~mm}$ bore silicone rubber tube filled with mercury and placed round the forearm. An increase in forearm circumference results in a change in electrical resistance in the mercury and this change can be calibrated to give the change in the arm circumference. Making the assumption that the arm is roughly cylindrical, the volume change can be calculated from the circumference change. Whitney has provided evidence that this method yields results which are very similar to the water-bath technique.

This strain gauge method was used for the experiment. It should not be forgotten that most patients view such procedures as to some extent stressful and, if valid estimates of relaxed blood flow are sought, the less elaborate the apparatus and the preparation the better. The setting up of the two cuffs and the strain gauge on the patient is a matter of not more than one or two minutes and is quite without discomfort, restricting the patient only to a very small degree.

In the present experiment the patient lay on a divan bed in a separate dimly illuminated and soundproofed room with all the recording apparatus isolated in an adjoining laboratory. The patient was observed through a one-way screen, a two-way intercom providing communication when necessary. Measurements were made on the left arm which was supported by pillows.

The sequence of relaxed and stressed measurements is shown in Fig. 2. This shows that each recording sequence begins with a 30 second rest period. The distal cuff then inflates to a pressure of $180 \mathrm{~mm}$ mercury. Fifteen seconds later the proximal cuff inflates to $80 \mathrm{~mm}$ mercury and the recording begins simultaneously. At each third recording from the sixth onwards the stress signal also commences

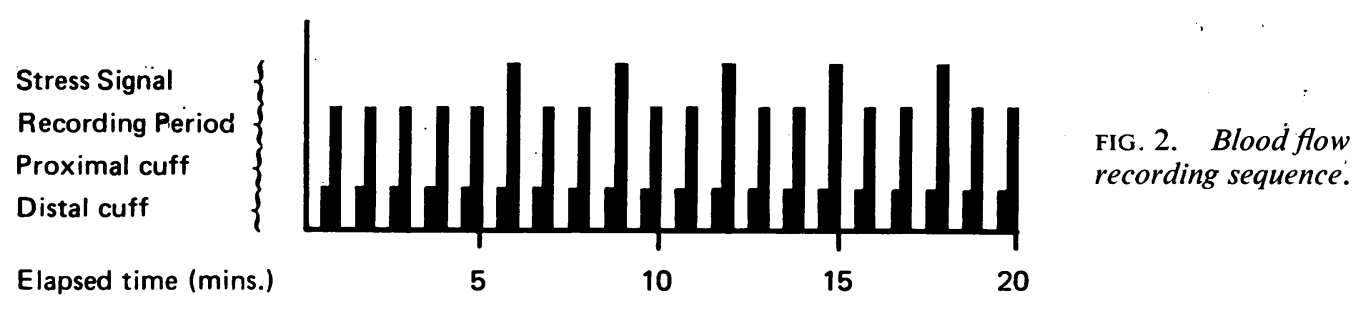


with the inflation of the upper cuff. The pressures employed were found by experiment to be the most generally satisfactory.

Thus 15 relaxing recordings and five stress recordings were obtained, as in Brierley (1969), but the total time was shortened from 30 to 20 minutes by limiting the actual recording time. The stresser used in the previous experiment was the amplified sound of a ticking clock but experiments showed that a pure tone of $1,000 \mathrm{~Hz}$ at approximately 80 decibels could be substituted. As such a signal was more readily reproduced it was adopted.

SUBJECTS The subjects were 19 depressed patients and 29 suffering from anxiety states. The mean age of the anxiety state patients was 31.6 years, $S D=12.8$ and the mean age of the depressed group was 38.7 years, $\mathrm{SD}=9 \cdot 7$. Again, as in the previous investigations, no evidence was found of a relationship between age and forearm blood flow within these groups; none of the blood flow parameters used correlated higher than 0.19 with age. The depressed group was composed of 11 males and eight females, and the anxiety group 10 males and 19 females.

The patients were referred by a number of departmental consultant psychiatrists and the clinical diagnosis was accepted as defining these two groups. No attempt was made to examine intra-group differences-for example, between generalized anxieties and phobias or between endogenous and reactive depressions. A wide range of types and degrees of anxiety and depression was included but all were regarded as materially disabled patients. I was not, of course, desirable to discontinue treatment for the present research so almost all subjects were currently taking psychotropic drugs of some kindes Many also would have night sedation prescribed The number and variety of such drugs was such as tô. prevent any meaningful analysis. However, patient? under treatment with electroconvulsive therapy wer avoided, although a number subsequently received? this treatment. The groups were, therefore, fairly representative of patients under treatment fo․ㅡ. depression or anxiety in an acute psychiatric unit.

The patients were informed of the research nature of the experiment and instructed to relax if possible They were forewarned that they would hear a noise like the whistle of a TV set and this was explained as being used to ensure that they were awake during the्ध recording. Patients who were severely disturbed o? clearly fearful of the experimental conditions wer excluded.

\section{RESULTS}

As shown by Brierley (1969), the forearm blo flow level for the anxiety group showed a tendency to fall throughout the recording sessigno If plotted as blood flow against log time sc this fall in blood flow appeared as a straight lîn

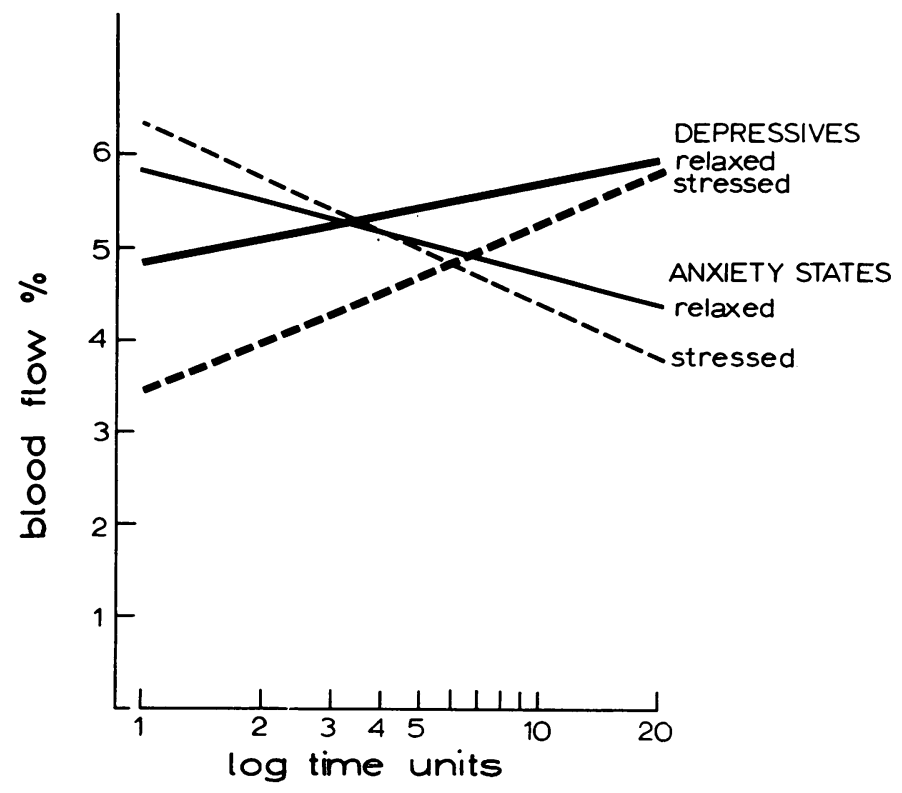

FIG. 3. Habituation curves of forearm blood flow in patients with anxiety states and depression. 
function. The linearity of the blood flow/log time function was assumed to hold for the present groups. In order to obtain the best fitting line on the relaxed and stressed values, the regression line coefficients were calculated. The regression line has the form $\mathrm{Y}=\mathrm{bX}+\mathrm{a}$, the regression coefficients being the ' $b$ ' and ' $a$ ' values. ' $a$ ' is the intercept value of the blood flow axis which amounts to the level of blood flow at the outset predicted from all the recordings. ' $b$ ' is the slope of the line, being an indication of the rate of fall of blood flow. A negative value of ' $b$ ' indicates that blood flow falls with time-that is, habituates-while the positive value shows the reverse-that blood flow increases or the patient becomes more aroused. Regression lines can be fitted to the relaxed recordings in two ways. They may be fitted to the first five relaxed recordings only, or to all 15 relaxed recordings. The objection to the latter calculation would be that the interspersed stressed recordings might interfere with the relaxed ones. Indeed, in the case demonstrated by Fig. 1, there will be some suggestion that this has occurred. However, the difference between the regression coefficients calculated for the first five recordings and those for the full 15 is very small. Table 1 shows this comparison for the separate groups. It therefore appears acceptable to consider all 15 recordings in calculating the relaxed values.

TABLE 1

REGRESSION COEFFICIENTS FROM FIRST FIVE COMPARED WITH ALL 15 RELAXED RECORDINGS

\begin{tabular}{lccccc}
\hline & \multicolumn{2}{c}{ Depression } & & \multicolumn{2}{c}{ Anxiety } \\
\cline { 2 - 3 } \cline { 5 - 6 } & 5 values & 15 values & & 5 values & 15 values \\
\hline $\begin{array}{c}\text { Initial level } \\
\text { ('a' coeff.) }\end{array}$ & 4.99 & 4.84 & & 5.61 & 5.57 \\
$\begin{array}{c}\text { Habituation } \\
\text { ('b' coeff.) }\end{array}$ & +0.74 & +0.74 & & -1.11 & -0.84 \\
\hline
\end{tabular}

Firstly, consider the lines for 'relaxed' blood flow only. Figure 3 shows that, under relaxed conditions, depressives and patients with anxiety states do not differ greatly in blood flow at the outset and this difference is far from statistical significance. Then, as the recordings continue, blood flow in the depressive group rises, which may be interpreted as an indication of increasing arousal. The blood flow in the patients with anxiety states, in comparison, falls or they become less aroused. However, neither the direction of change for each line nor the difference in the rate of change proves to be statistically significant, even though the result is consistent with the original hypothesis.

TABLE 2

RELAXED AND STRESSED MEASUREMENTS

\begin{tabular}{|c|c|c|}
\hline \multirow{2}{*}{$\begin{array}{c}\text { Forearm muscle blood flow } \\
\text { Mean } \\
\text { regression constants }\end{array}$} & \multicolumn{2}{|c|}{$\begin{array}{c}\text { Anxious and depressed patients } \\
\text { Diagnosis }\end{array}$} \\
\hline & Depression & Anxiety \\
\hline \multicolumn{3}{|l|}{ Relaxed values } \\
\hline ' $a$ ' coefficient & 4.99 & $5 \cdot 61$ \\
\hline 'b' coefficient & +0.74 & $-1 \cdot 11$ \\
\hline $\begin{array}{l}\text { Stressed values } \\
\text { 'a' coefficient } \\
\text { ' } b \text { ' coefficient }\end{array}$ & 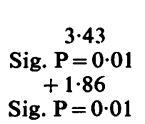 & $\begin{array}{r}6.31 \\
(t \text { test }) \\
-1.40 \\
(t \text { test })\end{array}$ \\
\hline
\end{tabular}

N.B.: ' $a$ ' coefficients represent intercept values on blood flow axis. ' $b$ ' coefficients represent slope, line showing habituation if - ve values, or arousal if + ve values.

Secondly, consideration of the 'stressed' lines shows a pattern similar to the 'relaxed' responses, except that the levels of blood flow change more rapidly. The blood flow of anxiety state patients falls more rapidly for the stressed recordings than the relaxed, while that for the depressives rises more rapidly. In these circumstances, the ' $b$ ' coefficients representing the slopes of the lines are significantly different tested by a $t$ test (Table 2). Sixty-nine per cent of the anxiety group show positive habituation or fall in blood flow, while $74 \%$ of the depressed group show increasing blood flow or increasing arousal under stress. Similarly, treating the groups separately, the change in blood flow in the anxiety group is shown to be significantly in the direction of a decrease $(P=0.03$ sign test $)$ and significantly in the direction of an increase for the depressives $(P=0.03$ sign test). In this instance, it is clear that the stressed blood flow 
change differentiates between the depressives and the patients with anxiety states.

Thirdly, one can examine the direction of the response to stress. The ' $a$ ' coefficients of the regression lines represent the initial level of blood flow predicted from the complete pattern of measurements throughout the session. Thus, the ' $a$ ' coefficients probably represent the best index of the initial level of blood flow under stress and under relaxation conditions and will indicate the direction of change of blood flow as a result of the stress signal and as the patient responds at the beginning of the recordings. Figure 3 suggests that the stress produces a fall in the forearm blood flow of depressives and a rise in anxiety states as was hypothesized. However, statistical tests of the direction of the change in blood flow from relaxed to stressed conditions assessed like this just failed to reach statistical significance. In the depressed group, $53 \%$ of subjects showed a fall in blood flow associated with stress, while only $28 \%$ of patients with anxiety states showed a fall in blood flow.

Finally, one may look at the initial blood flow levels as measured by regression ' $a$ ' coefficients. Under relaxed conditions the initial blood flows were not significantly different but again under stressed conditions the differing responses to stress result in a divergence of the initial blood flow levels of the two groups. Thus, these estimates of the initial stressed response show a level significantly higher in the anxiety group than in the depressive group as shown by a $t$ test (Table 2). Of course, as the measurements continue and the blood flow of one group falls and the other rises, this difference disappears as shown in Fig. 3.

\section{DISCUSSION}

The evidence presented here indicates differences between the patterns of forearm blood flow under stress exhibited by patients with anxiety states and depression. Interpretation of these differences is difficult at this stage. It cannot be said how far these features are transient sequelae of illness or how far they are representative of fundamental modes of adaptation of the subjects involved. The differences found may be permanent predispositions associated with the appearance of symptoms of depression or anxiety under stress, subjects tending to become over-aroused under stress suffering from anxiety states, while those who show a lessening of on arousal level suffer depression, possibly with retardation.

Previous work on psychophysiology has not $\stackrel{0}{2}$ reported this effect. However, close examination of other researches finds similar results obscured either by the particular physiological variables employed, the measurement techniques, or the underlying expectations in the experiment.

Dykman et al. (1968) have pointed to the fact that a field of research so apparently objective as psychophysiological measurement 'is remarkable for the results which are inconclusive, contradicting or paradoxical.... One of the most curious features in such researches is the consistency of the assumption that the subject not subjected to a controlled laboratory stress is, in fact, in a 'no stress' condition. This is manifestly untrue and the present results show variations of arousal leve to occur over a period of 20 minutes which arg not a direct result of the planned experiment寝음 stress. This being so, there is certainly no reasog to make the very common assumption that ab $\frac{D}{0}$ responses to a deliberately applied stress mus have the form of an increase in arousal. In fact $\overrightarrow{0}$ the response might be either a rise or a fall in the current level of arousal which might suggest a more complex repertoire of human stress response with 'flight' and 'freeze' characteristics. Clearly, the present result would lead one to expect conflicting research findings if the changes associated with adaptation to the experiment are not taken into account. No measurement of relaxation and stress response over a short period of time and neglecting the pattern of change is adequate. It, therefore, is far from simple to ensure that experiments in psychophysiology employing stress of any kind can be regarded as quite comparable. The full specification of the regression functions as here seems important at least.

In other fields, Foulds (1952) and Hetherington (1956) have provided some evidence that the disrupting of the 'flood of depressive thoughts' in the depressed patient improves his mental functioning. Foulds, for instance, showed that the tick of a metronome improved the test performance of depressives where perhaps the 
usual expectation would be that additional distraction would impede the problem solving. It is not inconceivable that, in the present experiment, the stresser also disrupted depressive thoughts and hence gave some measure of relief rather than stress.

The expectation of a one-way arousal effect has appeared in most researches. Duffy (1962), in her extensive review of physiological arousal, does not consider the occurrence or implications of falls in arousal level associated with stress. One reason for this is that a number of physiological variables frequently measured would not be suitable for the demonstration of increase and decrease of arousal in the same terms. Galvanic skin response (GSR) is perhaps the most common index of arousal employed. Darrow (1962) has pointed out that the recovery from a positive skin response is dependent on a variety of factors different from the initial response and hence is unlike the original response in form and rate. Spontaneous fluctuations of GSR as employed by Lader and Wing (1964; 1969), while showing that subjects with anxiety states have high fluctuation rates, show an almost complete absence of fluctuation in retarded depressives. Normal subjects show very few spontaneous fluctuations. Hence, this variable cannot adequately show a decrease in response to stress.

A number of researches show low levels of arousal to be associated with depression. Greenfield and Katz (1963) showed that subjects making small GSR responses to a $1,000 \mathrm{~Hz}$ tone were significantly more depressed according to the MMPI depression scale than the high responders. More to the point, this research shows clearly some or all of the depressed patients reacting to the tone to a lower degree than normal subjects. Gilbertstadt and Maley (1965) found that a stress produced a higher GSR in patients with anxiety states than in normal subjects and a lower than normal response in depressives. In this research, the authors selected the largest positive response (or the lowest conductance) in a given time after the stresser and so could have recorded responses only in one direction. Hattagandi et al. (1968) also considered only positive GSRs but again found that depressives (psychotic and neurotic) exhibited fewer GSRs and lower amplitude than did normal subjects.

Kelly and Walter (1968) show that non-agitated depressives have a forearm muscle blood flow that is below normal. However, Kelly adopts an unusual practice in taking the lowest recorded values as the best estimate of relaxed blood flow. The rationale of this convention seems reasonable were it not for the fact that the selected values will necessarily be those mostly affected by measurement error. It does also imply Kelly's assumption that responses to stress must be in the direction of blood flow increase.

Bagg and Crookes (1966) measured palmar sweat gland activity. They found that depressed women showed a lower sweat gland activity than normal subjects. So there is a fair consensus of research to suggest that depressives are in a lower state of arousal than the normal subjects and especially patients with anxiety states.

Investigations giving some hint of negative stress response are few. Ax (1953) and Schachter (1957) suggested distinct patterns of autonomic response associated with the action of adrenaline and noradrenaline. They distinguish these patterns as fear and anger in terms of the relative size of response. Schachter used the term 'peripheral resistance' as a measure of arousal. This was a measure of some complexity being blood pressure divided by an index of cardiac output. His results show a drop in 'peripheral resistance' in fear and an increase in anger. It is not possible to evaluate 'peripheral resistance' fully and the negative changes could have arisen from differing positive changes in the component measures. Nevertheless, Schachter's results are suggestive support for the present hypothesis.

On the present evidence, while clear differences emerge between clinically diagnosed anxiety and depressive groups, one cannot go further than point to the possibility of 'flight-freeze' response patterns. One cannot link these to forms of illness per se since they could arise from a number of idiosyncrasies in diagnosis or patient care. Roth et al. (1972) make a strong plea for rigour and objectivity in defining diagnostic groups in similar areas of research. 'Although problems in this field can be clarified by theoretical models and concepts they can be resolved only with the aid of observation on patients with affective disorders aimed at testing hypotheses with methods as rigorous as the situation permits'. Nevertheless, whatever proves to be 
the significance of a particular pattern of psychophysiological arousal characterizing a clinical group, it seems quite possible that it may be among the most objective features of that group.

\section{CONCLUSIONS}

The present paper shows that under the influence of a $1,000 \mathrm{~Hz}$ stress forearm muscle blood flow differentiates groups of mixed depressives and anxiety states. The depressives at the outset show lower blood flow values but, as the measurement session continues, these rise, while those of the patients with anxiety states fall. There is a suggestion that depressives show a fall in blood flow from their non-stressed level, in response to the stress, compared with the increase shown by those with anxiety states. These differing patterns illustrate the possibility of flight-freeze patterns of stress response in clinical subjects.

The whole investigation emphasizes the need for precise delineation of diagnostic groups as well as the impracticability of properly defining levels of arousal by psychophysiological methods unless the variation over a material period of time is considered.

\section{REFERENCES}

Ax, A. F. (1953). The physiological differentiation between fear and anger in humans. Psychosomatic Medicine, 15, 433-442.

Bagg, C. E., and Crookes, T. G. (1966). Palmar digital sweating in women suffering from depression. British Journal of Psychiatry, 112, 1251-1255.

Brierley, H. (1969). The habituation of forearm muscle blood flow in phobic subjects. Journal of Neurology, Neurosurgery, and Psychiatry, 32, 15-20.

Darrow, C. W. (1962). The rationale for treating the change in
GSR as a change in conductance. Paper read to Symposium on Problems in Electrodermal Measurement. American Psychological Association, St. Louis.

Dykman, R. A., Reese, W. G., Galbrecht, C. R., Ackerman, P. T., and Sundermann, R. S. (1968). Autonomic responses in psychiatric patients. Annals of the New York Academy of Science, 147, 237-303.

Duffy, E. (1962). Activation and Behavior. Wiley: New York.

Foulds, G. A. (1952). Temperamental differences in maze performance. Part 2. British Journal of Psychology, 43, $33-41$.

Gilberstadt, H., and Maley, M. (1965). GSR, clinical state and psychiatric diagnosis. Journal of Clinical Psychology, $\vec{\Phi}$ 21, 235-238.

Greenfield, N. S., Katz, D., Alexander, A. A., and Roessler, R. (1963). The relationship between physiological and psychological responsivity: depression and the galvanic skin response. Journal of Nervous and Mental Disease, 136, 535-539.

Hattagandi, S., Lidsky, A., Lee, H., and Ban, T. A. (1968). Orienting-reflex behavior and clinical psychopathology. Conditional Reflex, 3, 29-33.

Hetherington, R. (1956). The effects of E.C.T. on the efficiency and retentivity of depressed patients. British Journal of Medical Psychology, 29, 258-269.

Jamieson, R. (1970). Orienting reflexes and the stress response in the measurement of anxiety. Paper read to London Conference of British Psychological Society.

Kelly, D. H. W. (1966). Measurement of anxiety by forearnp is blood flow. British Journal of Psychiatry, 112, 789-798.

Kelly, D. H. W., and Walter, C. J. S. (1968). The relationshig f between clinical diagnosis and anxiety, assessed by forear blood flow and other measurements. British Journal $\mathscr{\&}$ Psychiatry, 114, 611-626.

Lader, M. H., and Wing, L. (1964). Habituation of the psycho-galvanic reflex in patients with anxiety states and i⿱ normal subjects. Journal of Neurology, Neurosurgery, a Psychiatry, 27, 210-218.

Lader, M. H., and Wing, L. (1969). Physiological measures 을 agitated and retarded depressed patients. Journal of Psychiatric Research, 7, 89-100.

Lader, M. H., and Mathews, A. M. (1968). A physiological model of phobic anxiety and desensitization. Behaviour Research and Therapy, 6, 411-421.

Roth, M., Gurney, C., Garside, R. F., and Kerr, T. A. (1972). Studies in the classification of affective disorders. British Journal of Psychiatry, 121, 147-161.

Schachter, J. (1957). Pain, fear, and anger in hypertensives and normotensives. Psychosomatic Medicine, 19, 17-29.

Whitney, R. J. (1953). The measurement of volume changes in human limbs. Journal of Physiology, 121, 1-27. 